\title{
New Metamaterial Absorber Based on Electromagnetic Coupling of Triangular SRR and Square SRRs for X-Band RADAR Applications
}

MOHAMMED BERKA ( $\sim$ mohammed_76_berka@yahoo.fr )

University of Mascara

\section{Driss Bouguenna}

Mustapha Stambouli Mascara University: Universite Mustapha Stambouli Mascara

Amina Bendaoudi

University of Sidi Bel Abbes

Zoubir Mahdjoub

University of Sidi Bel Abbes

\section{Research Article}

Keywords: Absorber, Metamaterial, Permeability, Split Ring Resonators (SRRs), Transverse Electric (TE)

Posted Date: June 9th, 2021

DOl: https://doi.org/10.21203/rs.3.rs-504675/v1

License: (c) (i) This work is licensed under a Creative Commons Attribution 4.0 International License.

Read Full License 


\title{
New Metamaterial Absorber Based on Electromagnetic Coupling of
}

\section{Triangular SRR and Square SRRs for X-Band RADAR Applications}

\author{
Mohammed Berka ${ }^{1,2, *}$, Driss Bouguenna ${ }^{3}$, Amina Bendaoudi ${ }^{2}$, and Zoubir Mahdjoub ${ }^{2}$ \\ 1) Electrical Engineering Department, Science and Technology Faculty, Mustapha Stambouli University of Mascara, Mascara \\ 29000, Algeria. \\ 2) EPO Laboratory, Electronic Department, Sidi Bel Abbès University, Sidi Bel Abbès 22000, Algeria. \\ 3) Geomatics, Ecology and Environment Laboratory, Nature and Life Sciences Faculty, and Department of Science and \\ Technology, Science and Technology Faculty, Mustapha Stambouli University of Mascara, Mascara 29000, Algeria.
}

Abstract - In this paper, a new metamaterial absorber (MA) is presented. The proposed absorber is a microwave structure constituted by a network of four split ring resonators (SRRs) of magnetic resonance and negative permeability $(\mu<0)$; one resonator SRR central for triangular shape and three others resonators SRRs of the same square shape and the same dimensions. All resonators SRRs are printed on the upper surface of the FR4_Epoxy substrate $\left(\varepsilon_{r}=4.4 ; \operatorname{tg} \delta=0.02\right)$, its dimensions are chosen to obtain the resonance in the X-band frequency. To eliminate the transmission, we add a copper conductive metal plate that will be etched on the upper face of the same substrate. The proposed MA is optimized for dimensions of $(27.9 \mathrm{~mm} \times 32 \mathrm{~mm}$ ), its electromagnetic characteristics are studied for transverse electric (TE) polarization for different incidences and different inter-resonator spacing. The obtained results with the simulations performed by the high-frequency structure simulator (HFSS) show that our metamaterial absorber still has three peaks of different absorptions estimated at a maximum on the order of $98.76 \%$. The main advantage of this study based on the proposed structure is that it is possible to control its absorption percentage for $\mathrm{X}$-band radar applications.

Keywords - Absorber, Metamaterial, Permeability, Split Ring Resonators (SRRs), Transverse Electric (TE). 
* Corresponding author: e-mail address: mohammed_76_berka@yahoo.fr or m.barka@univ-mascara.dz (Mohammed Berka)

\section{INTRODUCTION}

During the last decade, wireless communication systems using microwave devices have undergone remarkable development. The study of most microwave devices requires a remarkable knowledge of the physical characteristics of electromagnetic waves when they propagate in structures. These electromagnetic characteristics are often reflection, transmission, refraction, radiation, diffraction and also absorption [1-5]. The absorption of electromagnetic (EM) waves has been the subject of several recent studies [6-9], the control of the absorption percentage is becoming more and more essential to meet the needs of absorber performance. Nowadays, metamaterials absorbers offer a prestigious position in the world of telecommunications, especially for antennas [10] and radars [11].

Metamaterials are artificially designed materials with different properties than natural materials. This kind of medium was introduced for the first time by Victor Veselago [12] in 1967 after the Second World War. He showed that the propagation of electromagnetic waves in metamaterials is carried out in the opposite direction to that of natural materials. Previously, left-handed metamaterials, whose permittivity and permeability are negative [13] have been a favorite topic for millimeter-wave and for microwave societies because of their unique unusual phenomena, such as negative index refraction [14] and the generation of surface plasmon.

In this work, we propose a new metamaterial absorber (MA) consisting of four split ring resonators (SRRs). The overall shape of our absorber is based on a central resonator SRR with triangular shape surrounded by three resonators SRRs of the same square shape and also of the same dimensions (engraved on the upper face of the used substrate) in order to obtain resonances in the X-band. These square resonators SRRs are spaced apart by an inter-resonator distance $(e)$, one of these three resonators SRRs is coupled to an etched copper conductive plate to prevent transmission of the overall structure. The polarization of our MA 
is TE for different incidences and the study is based on electromagnetic coupling of triangular resonator SRR to square resonator SRRs to have different absorption levels in the same X-band frequency.

\section{METAMATERIAL ABSORBER DESIGN PROCEDURE}

\section{II.1. DEPICTION}

The resonator SRR is a metamaterial resonator proposed for the first time by J. Pendry and his research team [15]. Geometrically, the resonator SRR is formed by two outer and inner split rings with two opposing interrupts to have the capacitive effect. Physically, the resonator SRR can support too small wavelengths; see lengths of the order of a few microns [16].

\section{II.1.1. TRIANGULAR SPLIT-RING RESONATOR}

The triangular resonator SRR of period $P_{1}$ is a metamaterial resonator formed by two inner and outer rings. These two rings have a triangular shape (equilateral triangle) of $a$ and $b$ ribs for the outer and inner ring, respectively. All interrupts of the rings have the same value which is $g_{t}$, the width $W$ is the same for both rings. The triangular resonator SRR is represented by Fig. 1.

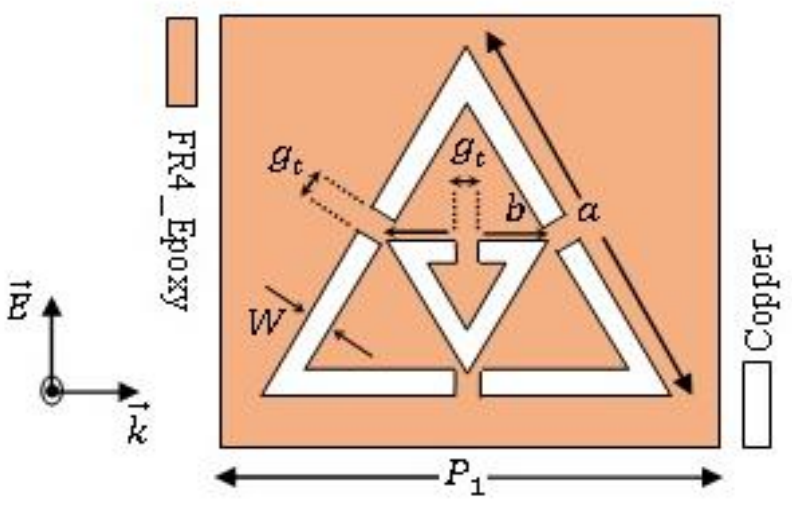

Fig. 1 Triangular resonator SRR

The proposed triangular resonator SRR dimensions are shown in Table I.

Table I. Triangular resonator SRR dimensions.

\begin{tabular}{cccccc}
\hline Parameter & $a$ & $b$ & $g_{t}$ & $W$ & $P_{1}$ \\
\hline Value $(\mathrm{mm})$ & 6.4 & 2.64 & 0.2 & 0.2 & 7.2 \\
\hline
\end{tabular}


The study of the elementary components of a metamaterial resonator shows that the origin of the resonance comes mainly from the Lorentz dispersion [17], which led us to propose an equivalent electrical scheme for each resonator, this scheme is based on a resonant circuit.

The equivalent electrical circuit of the triangular resonator SRR is composed of three resonators each one formed by an inductance $L_{1}$ and a capacitance $C_{1}$, these three resonators represent the outer ring of our triangular resonator SRR. The inner ring corresponds to a resonator formed by an inductance $L_{2}$ and a capacitance $C_{2}$. The capacitance $C_{p}$ represents the coupling between the two inner and outer rings of the triangular resonator SRR. Fig. 2 shows the equivalent circuit model of the triangular resonator SRR.

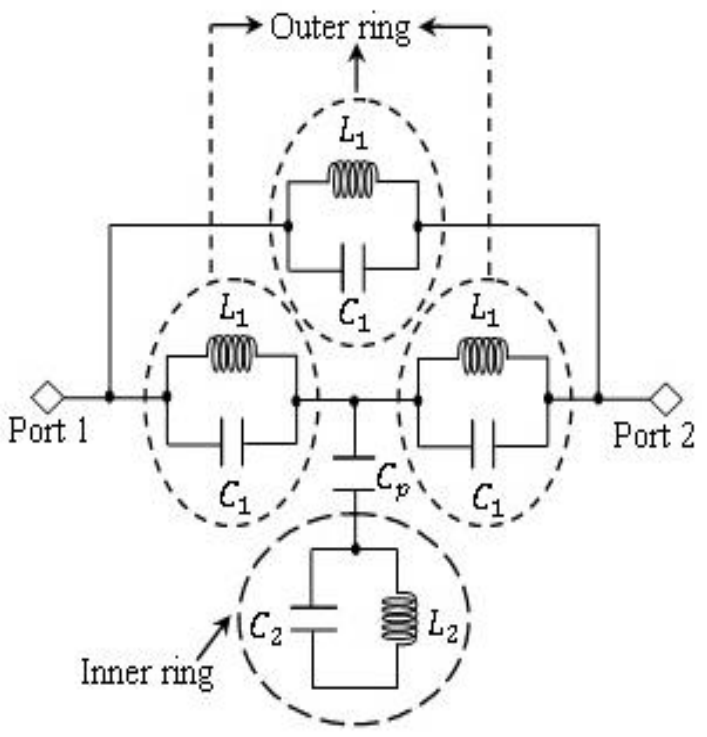

Fig. 2 Triangular resonator SRR equivalent electrical circuit

\section{II.1.2. SQUARE SPLIT-RING RESONATOR}

As triangular resonator SRR, the square resonator SRR is also formed by two rings; inner of radius $R_{1}$ and outer of radius $R_{2}$ for square shape. The period of the proposed square resonator SRR is $P_{2}, l$ represents the spacing between the two rings that have the same width $d$, the interruption gaps $g_{s}$ have the same value as the interrupt $g_{t}$ for the triangular resonator SRR. 
The square resonator SRR and its equivalent circuit model are shown in Fig. 3.

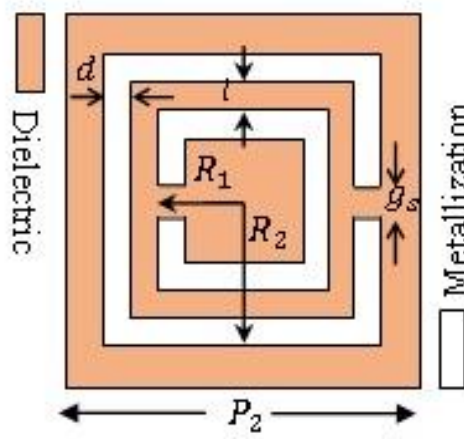

(a)

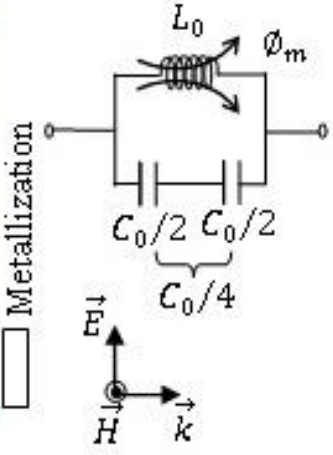

(b)

Fig. 3 Square resonator SRR; a) representation in rings and b) equivalent circuit model

The proposed square resonator SRR dimensions with $(l=d)$ are shown in Table II.

Table II. Square resonator SRR dimensions.

\begin{tabular}{cccccc}
\hline Parameter & $g_{s}$ & $d$ & $R_{1}$ & $R_{2}$ & $P_{2}$ \\
\hline Value $(\mathrm{mm})$ & 0.2 & 0.2 & 2.8 & 3.2 & 6.8 \\
\hline
\end{tabular}

The square resonator SRR can be mainly considered as a magnetic dipole, its equivalent circuit model behaves like a $L_{0} C_{0}$ resonator excited by a magnetic field perpendicular to the plane of the rings [18]. The equivalent circuit model of the square resonator SRR is shown in Fig. 3 b.

\section{II.2. PROPOSED METAMATERIAL ABSORBER}

Our proposed metamaterial absorber (MA) consists of four metamaterial resonators SRRs; three resonators SRRs of square shapes (for dimensions shown in Table II) centered on a triangular resonator SRR (for dimensions shown in Table I) and separated from each other by an angle $\left(\varphi=120^{\circ}\right)$. These resonators; triangular and squares are engraved on the upper face of the used substrate, a metal plate (of copper) is etched on the upper face of the same substrate to prevent the transmission of the overall structure.

The dimensions of this plate are optimized at $\left(a_{1}=a=2 R_{2}=6.4 \mathrm{~mm}\right.$ and $\left.W_{1}=1.5 \mathrm{~mm}\right)$. We polarize the metamaterial absorber proposed to have the TE polarization and we will study the absorption as a function of the inter-resonator electromagnetic coupling distance $(e)$ between each square resonator SRR and the 
central triangular resonator SRR. Then, we will study the absorption according to the numerical values of the incidence angle $(\theta)$. The optimized dimensions of the proposed MA are $(m \times n) \mathrm{mm}^{2}$ (with $m=27.9 \mathrm{~mm}$ and $n=32 \mathrm{~mm}$ ). Fig. 4 represents our proposed MA.

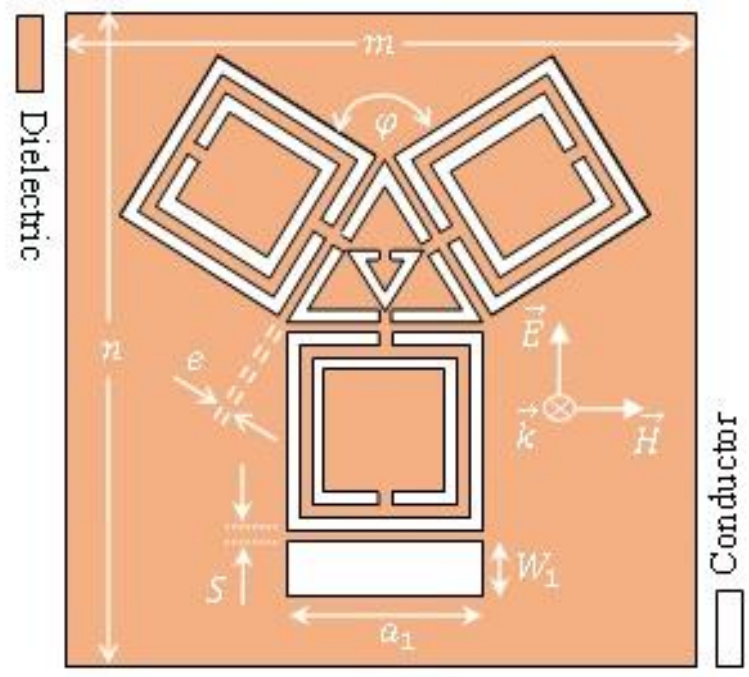

(a)

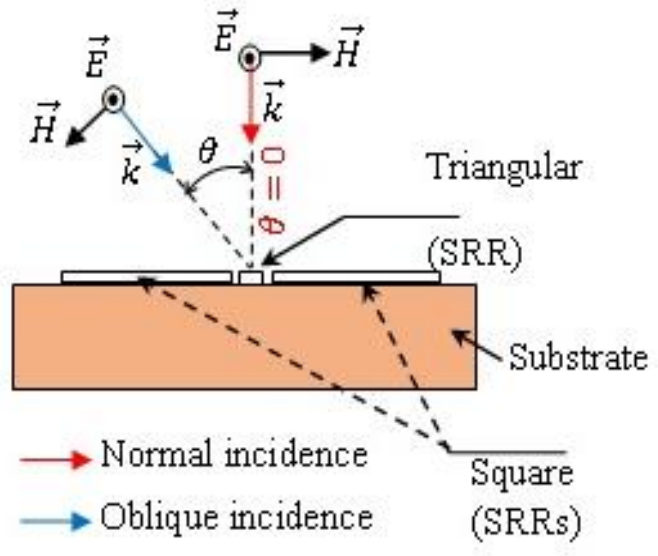

(b)

Fig. 4 Proposed MA for TE polarization. a) Top view and b) Side view (from the second port).

\section{SIMULATION RESULTS AND DISCUTION}

\section{III.1. TRIANGULAR SPLIT-RING RESONATOR BEHAVIOR}

On the upper surface of the FR4_Epoxy substrate $\left(\varepsilon_{r}=4.4\right.$ and $\left.t g \delta=0.02\right)$, of thickness $(h=1.53 \mathrm{~mm})$, the triangular metamaterial resonator SRR is printed for a thickness $(t=15 \mu \mathrm{m})$. It is represented in the 3-D Modeler of the HFSS simulator, as shown in Fig. 5. 


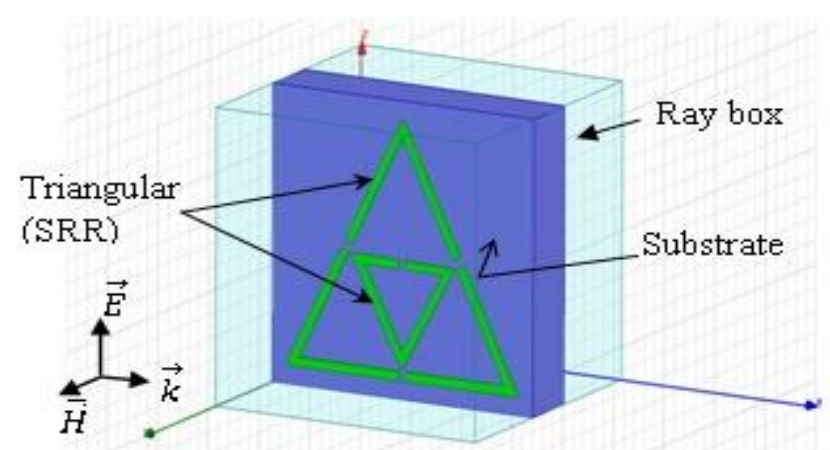

Fig. 5 Triangular resonator SRR polarization according to $(O Z)$

On the simulator, we polarize the triangular resonator SRR in the direction $(O Z)$ and we set the boundary conditions on the fields; electric and magnetic, respectively. The module and the phase of the $S_{11}$ parameter are shown in Fig. 6.

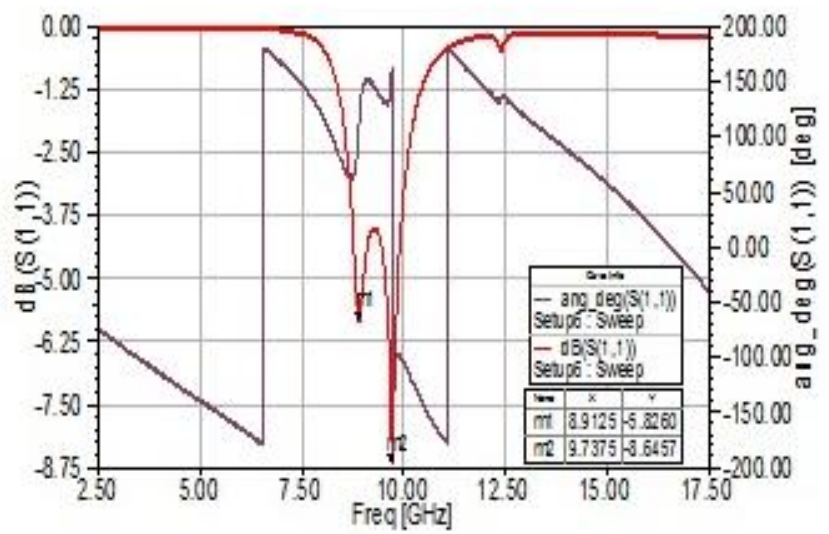

Fig. $6 S_{11}$ Parameter of triangular resonator SRR, module and phase

Fig. 6 shows the behavior of the triangular resonator SRR based on its $S_{11}$ parameter (module and phase). For the amplitude of $S_{11}$, we note that it is about two resonance frequencies in the X-band [8-12] GHz; the frequency $f_{r 1}=8.91 \mathrm{GHz}$ of the low resonance associated with the inner ring of the triangular resonator SRR and the frequency $f_{r 2}=9.73 \mathrm{GHz}$ of the high resonance associated with the outer ring. For the $S_{11}$ argument, we notice that the values vary in the interval $[-\pi,+\pi]$. 
The triangular resonator SRR has characteristic impedance $Z(\omega)$ defined by the relation.

$Z(\omega)=\sqrt{\frac{\mu_{r}(\omega)}{\varepsilon_{r}(\omega)}} Z_{0}$

Where $\mu_{r}(\omega)$ and $\varepsilon_{r}(\omega)$ represent the relative permeability and the relative permittivity of the triangular resonator SRR, respectively and $Z_{0}$ represents the characteristic impedance of the vacuum $\left(Z_{0} \approx 377 \Omega\right.$.) We note that the triangular resonator is adapted for $\left(Z(\omega)=Z_{0}\right)$.

The effective reduced impedance of the triangular resonator SRR is defined by [19].

$$
Z_{e f f}(\omega)=\frac{Z(\omega)}{Z_{0}}=\sqrt{\frac{\left(1+S_{11}(\omega)\right)^{2}-S_{21}^{2}(\omega)}{\left(1-S_{11}(\omega)\right)^{2}-S_{21}^{2}(\omega)}}
$$

For a zero transmission $\left(S_{21}(\omega)=0\right)$, then

$Z_{e f f}(\omega)=\sqrt{\frac{\left(1+S_{11}(\omega)\right)^{2}}{\left(1-S_{11}(\omega)\right)^{2}}}$

And also,

$Z(\omega)=\operatorname{Re}\{Z(\omega)\}+i \operatorname{Im}\{Z(\omega)\}=Z^{\prime}(\omega)+i Z^{\prime \prime}(\omega)$

In the HFSS simulator, we directly select both parts; real (with $Z^{\prime}(\omega)>0$ ) and imaginary characteristic impedance. Fig. 7 shows the variation of these two impedance parts.

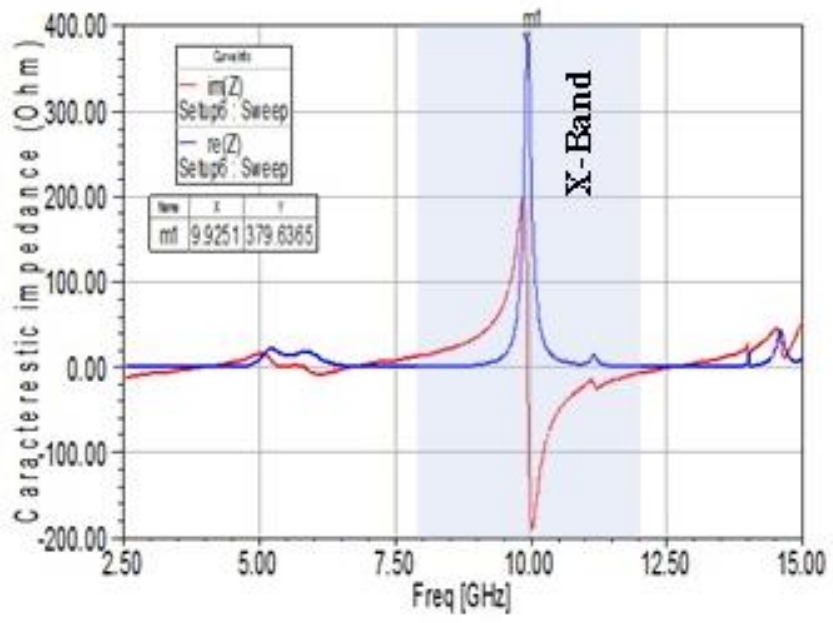

Fig. 7 Real and imaginary parts of the characteristic impedance of the triangular resonator SRR 
As shown in Fig. 7, at resonance frequency, the real part of the characteristic impedance of the triangular resonator SRR approaches to the vacuum impedance (i.e. approximately $377 \Omega$ ) while the imaginary part tends to 0 . So, the energy that is not reflected is trapped and absorbed into the resonator. The triangular resonator SRR absorption is given by [20].

$$
A_{b}=1-R-T=1-\left|S_{11}(\omega)\right|^{2}-\left|S_{21}(\omega)\right|^{2}
$$

Where $R=\left|S_{11}(\omega)\right|^{2}$ and $T=\left|S_{21}(\omega)\right|^{2}=0$.

So,

$$
A_{b}=1-\left|S_{11}(\omega)\right|^{2}
$$

Fig. 8 shows the absorption $\left(A_{b}\right)$ and the reflection $(R)$ of the triangular resonator SRR.

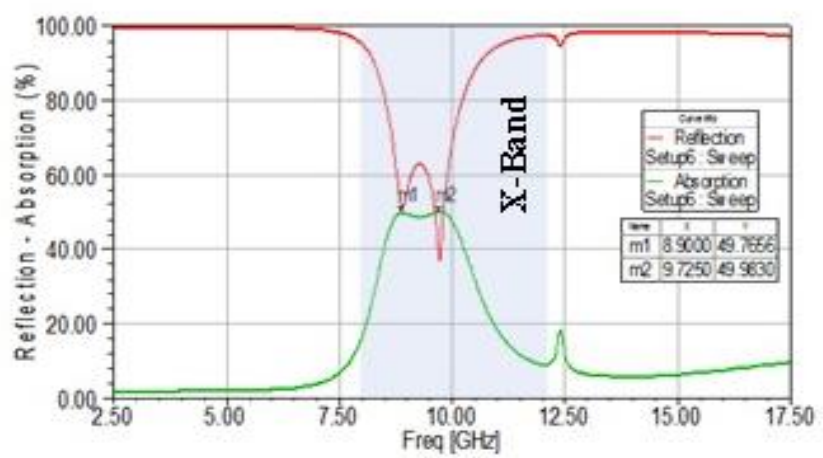

Fig. 8 Reflexion and absorption of the triangular resonator SRR

Reflection and absorption of the triangular resonator SRR are shown in Fig. 8. We note that at the two magnetic resonances of the triangular resonator SRR $\left(f_{r 1}=8.91 \mathrm{GHz}\right.$ and $\left.f_{r 2}=9.73 \mathrm{GHz}\right)$, we have the two absorptions $A_{b_{1}}=49.76 \%$ and $A_{b_{2}}=49.98 \%$, respectively. It can also be noted that the absorption of this resonator SRR is modest, where the biggest is associated with the smallest reflection $-8.64 \mathrm{~dB}$.

\section{III.2. SQUARE SPLIT-RING RESONATOR BEHAVIOR}

On the upper surface of the same substrate used for the triangular resonator SRR, the square metamaterial resonator SRR is etched for the same thickness $(t=15 \mu \mathrm{m})$. Fig. 9 shows this resonator in 3-D Modeler of HFSS. 


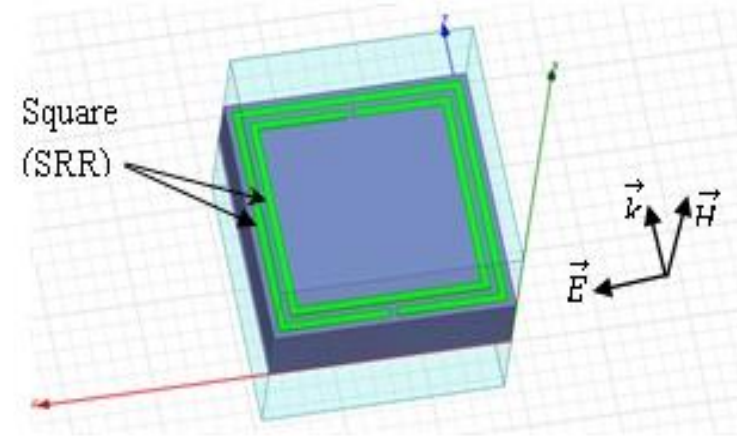

Fig. 9 Square resonator SRR polarization according to $(O Z)$

The reflection and the absorption of square resonator SRR are shown in Fig. 10.

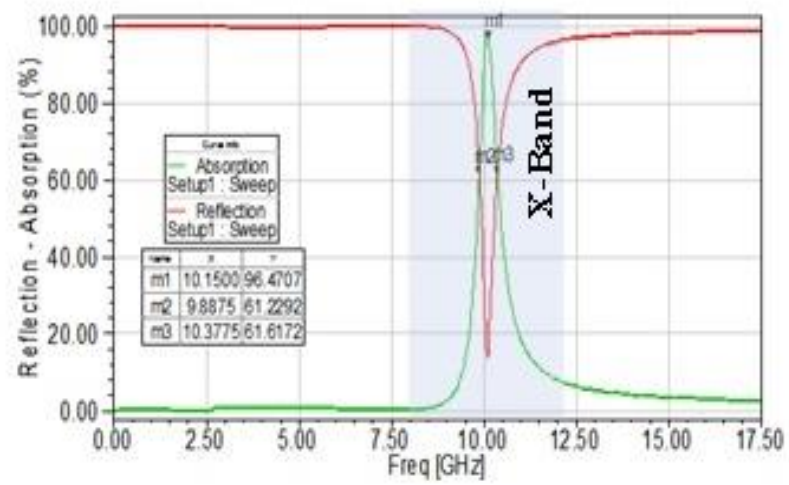

Fig. 10 Reflection and absorption of square resonator SRR

In Fig. 10, we note that there is one resonance (in the $\mathrm{X}$-band) associated with the frequency $f_{r}=10.15 \mathrm{GHz}$, the maximum absorption estimate of this resonator SRR at the indicated resonance frequency is $A_{b}=96.47 \%$ with a thickness of the order of $(\lambda / 19)$. It is also noted that the absorption is obtained for a narrow band of the order of $0.49 \mathrm{GHz}$.

\section{III.3. ELECTROMAGNETIC CHARACTERISTICS OF THE PROPOSED (MA)}

To simulate the proposed MA consisting of three square resonators SRRs and a central triangular resonator SRR, we fixed simulation criteria which will be adapted to complicated microwave structures. So, two Master-Slave boundary conditions are used along the $\vec{E}$ and $\vec{H}$ axes. To generate the incident wave (designed along the $\vec{k}$ axis) on the surface of our MA, we must use a Floquet-port that is assigned to the 
upper limit of the structure. The lower limit of the overall structure is indicated by the lower surface of the substrate (since a metal plate has been used on the upper surface of the substrate to prevent transmission). Fig. 11 shows the simulation model in three dimensions of our proposed MA integrated in HFSS simulator.

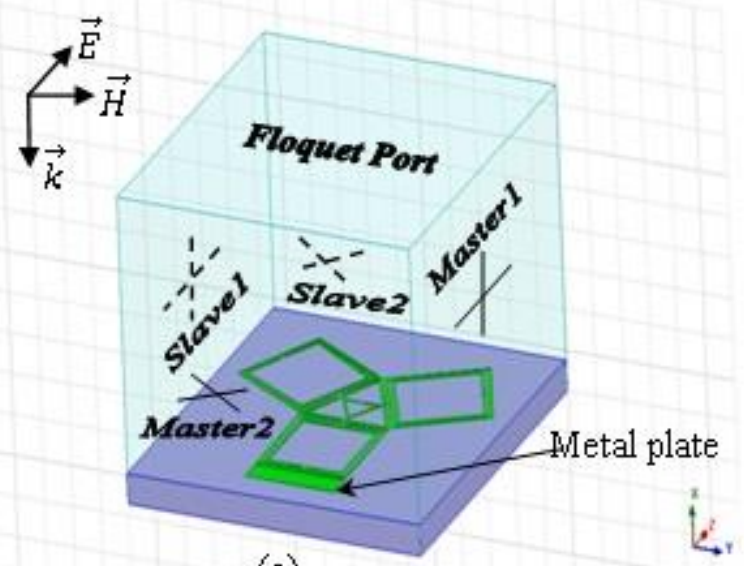

(a)

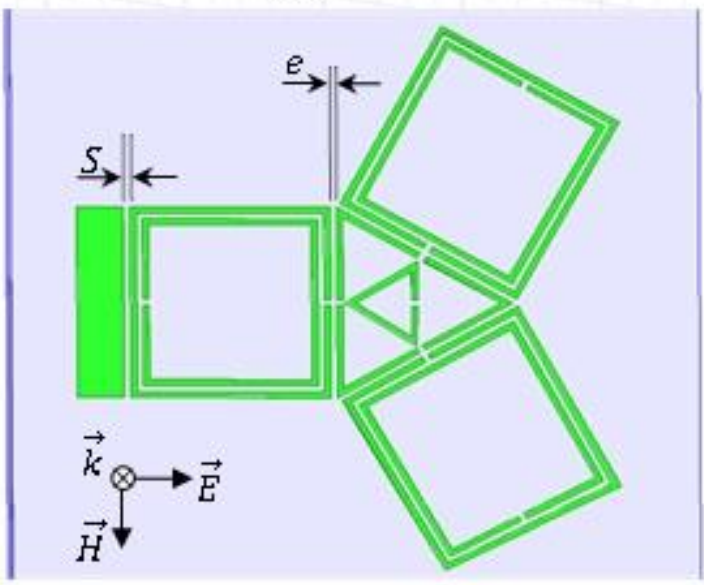

(b)

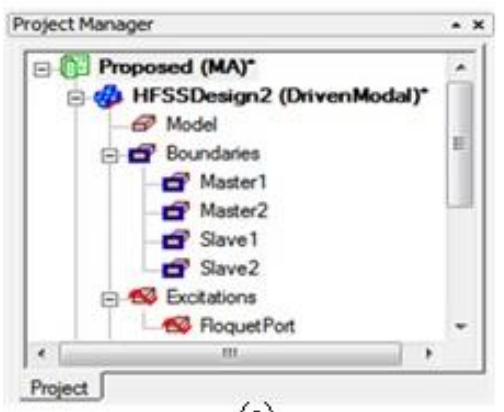

(c)

Fig. 11 The proposed MA; a) 3-D simulation model; b) Top view; and c) Project Manager 
According to the inter-resonator spacing value $(e=0.1 \mathrm{~mm}, e=0.2 \mathrm{~mm}, e=0.3 \mathrm{~mm})$, we have done three simulations for the proposed MA for normal incidence and a fixed value of the spacing between the metal plate and one of the three square resonators SRRs $(S=0.2 \mathrm{~mm})$. Then, we searched the absorption of the proposed MA for the TE polarization, also for the normal incidence $\left(\theta=0^{0}\right)$ and for three oblique incidence angle $\left(\theta=30^{\circ}, \theta=45^{\circ}\right.$ and $\left.\theta=60^{\circ}\right)$.

The absorption of our MA according to the inter-resonator spacing and the incidence angle (for TE polarization) is shown in Fig. 12 and Fig. 13, respectively.

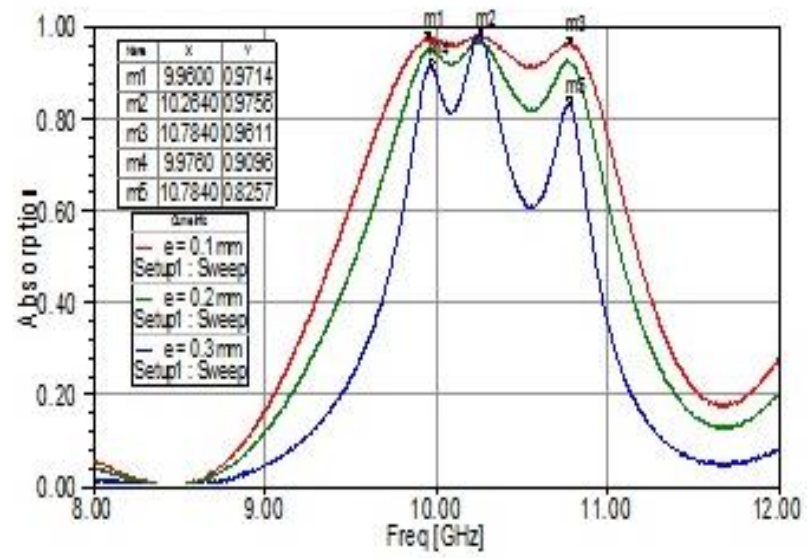

Fig. 12 Absorption of proposed MA according to the inter-resonator spacing

In Fig. 12, we note that our proposed metamaterial absorber has three absorption peaks in the X-band. The three absorption peaks are relative to the three squares resonators SRRs. For $e=0.1 \mathrm{~mm}$, we have the three absorptions $97.14 \%, 97.56 \%$ and $96.11 \%$ for the three resonances $9.96 \mathrm{GHz}, 10.26 \mathrm{GHz}$ and 10.78 GHz, respectively. We also note that the most important absorption among the three indicated, is that which is around the resonance of the square resonator SRR (previously estimated of the order of $96.47 \%$ around $10.15 \mathrm{GHz}$ ). It is noted in the same figure that the absorption of the proposed metamaterial absorber increases when the value of the spacing $e$ decreases, which proves that the absorption is greater for a considerable electromagnetic coupling between the metamaterial resonators in the absorber. 


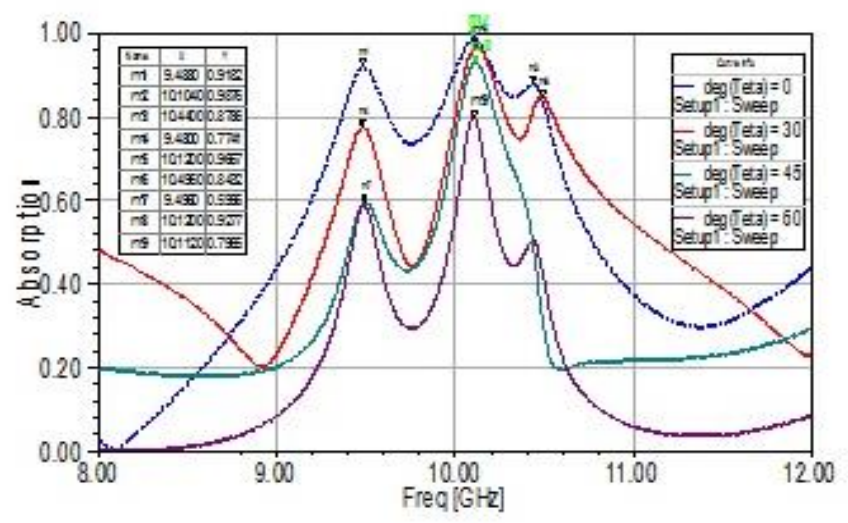

Fig. 13 Absorption of proposed MA according to the angle of incidence

Fig. 13 shows the simulated absorption of the proposed metamaterial absorber in the X-band for normal incidence $\left(\theta=0^{0}\right)$ and oblique (for three values of $\theta$ ). We notice in this Figure that the absorption has always three peaks. For the normal incidence, it is noted that the absorption is estimated by the highest percentage $98.76 \%$ at the frequency $10.19 \mathrm{GHz}$ (around the resonance of the square resonator SRR, the other two peaks represent the two absorptions estimated by $91.82 \%$ and $87.86 \%$ at the resonances $9.48 \mathrm{GHz}$ and $10.44 \mathrm{GHz}$, respectively. We note also that the absorption for the TE polarization decreases as the angle of incidence increases, the absorptions around the resonance of the square resonator SRR are estimated at 92.77\% $\left(\theta=45^{\circ}\right)$ and $79.65 \%\left(\theta=60^{\circ}\right)$ at $10.12 \mathrm{GHz}$ and $10.11 \mathrm{GHz}$ frequencies, respectively. Fig. 14 can shows this decrease in absorption when the incidence angle increases.

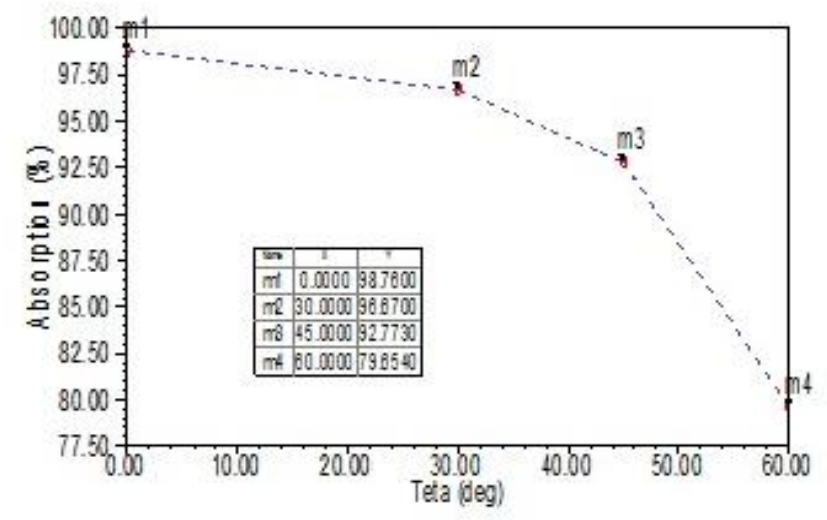

Fig. 14 Absorption points for selected incidences.

The electric field mapping for the proposed MA at the central resonance (for the normal incidence) is represented by Fig. 15. 


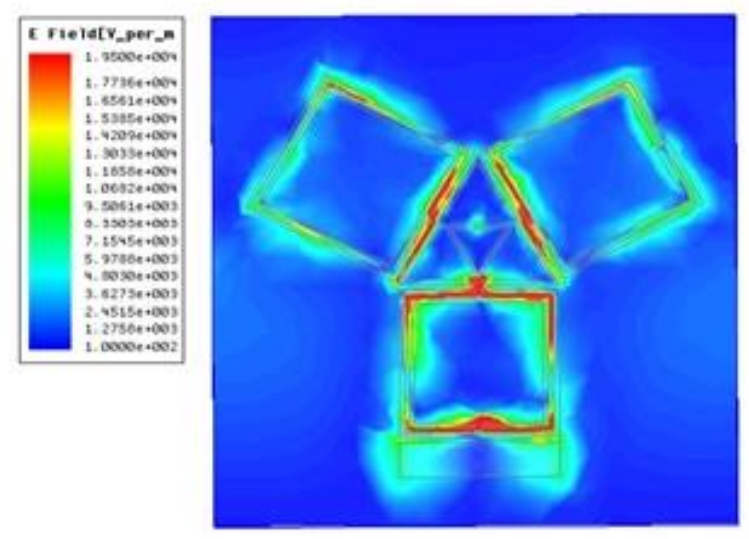

Fig. 15 Mapping of the electric field in the proposed MA at $10.26 \mathrm{GHz}$ (for $e=0.2 \mathrm{~mm}$ )

On Figure 15 we note that the electric field at the resonance of $10.26 \mathrm{GHz}$ is more condensed in the regions situated between the triangular resonator SRR and the square resonators SRRs, which justifies the effect of the electromagnetic coupling on the absorption of the structure. In the vicinity of the gaps of each metamaterial resonator constituting the absorber, it is also noted that the electric field can take considerable amplitudes which shows the capacitive effect of the resonators SRRs; triangular and squares in these region.

\section{CONCLUSIONS}

In this work, we have proposed a new microwave structure that is a MA. Our proposed MA is based on the electromagnetic coupling between three resonators SRRs for one central triangular and square shape resonators. All the dimensions of these metamaterial resonators are chosen to have magnetic resonances in the X-band and thus to have the necessary absorption in the same band. We used a printed metal plate on the upper face of the used substrate to prevent transmission of the structure. We polarized our MA to have the TE polarization for different angles of incidence. During our study, we also varied the inter-resonator spacing. In our results, we have always found three absorption peaks relative to the coupling of the triangular resonator to the three square resonators where the biggest appeared around the resonance of each square resonator for the normal incidence. Based on these results, our proposed MA may represent the base cell for several future RADAR applications systems in the frequency X-Band. 


\section{ACKNOWLEDGEMENTS}

We express our sincere thanks to the General Directorate of Scientific Research and Technological Development (DGRSDT) for their support in the development of this work.

\section{CONFLICT OF INTEREST}

The authors declare that they have no conflict of interest.

\section{REFERENCES}

1. Amri, H., Zaabat, M.: Electromagnetic Modeling of Active Circuit using Wave Concept Iterative Process. Bulletin of Elec Eng and Inf. 5 (2), 187-193 (2016)

2. Šmichrast, L.: On Some Aspects of the Complex-envelope Finite-differences Simulation of Wave Propagation in One-dimensional Case. Journal of ELECTRICAL ENGINEERING, 65(5), 265-270 (2014)

3. Elwasif, K., Abuishaiba, S., Shabat, M.: 3D-FDTD Head Model Exposure to Electromagnetic Cellular Phones Radiation. American Journal of Electromagnetics and Applications, Sciences Publishing groupe. 6(2), $42-48(2018)$

4. Foteinopoulou, S., Kafesaki, M., Economou, E., Soukoulis, C. : Backward surface waves at photonic crystals. Phy Rev B. (2007)

5. Tremain, B., Hooper, I., Sambles, J., Hibbins, A.: Isotropic Backward Waves Supported by a Spiral Array Metasurface. Scientific Reports, Article DOI:10.1038/s41598-018-25469-7 (2018)

6. Devin, K., Borcov, A., Devina, E.: Gradient Absorber of Electromagnetic Waves. Inorganic Materials: Applied Research, 10 (2), 459-462 (2019)

7. Feng, J., et al.: Polarization-Independent and Angle-Insensitive Metamaterial Absorber Using 90Degree-Rotated Split-Ring Resonators. International Journal of Antennas and Propagation, Hindawi Publishing Corporation, Article ID 240691 (2015) 
8. Kenanakis, G., et al.: Perfect absorbers based on metal-insulator-metal structures in the visible region: simple approach for practical applications. Applied Physics A, Materials Science \& Processing (2017)

9. Hossain, M., Faruque, M., Islam, M., Mat, K. : A New Compact Octagonal Shape Perfect Metamaterial Absorber for Microwave Applications. Applied Sciences (2017)

10. Ren, J., Gong, S., Jiang, v.: Low-RCS Monopolar Patch Antenna Based on Dual-Ring Metamaterial Absorber. IEEE Antennas and Wireless Propagation Letters (2017)

11. Zhong, S., Wu, L., Liu, T., Huang, J., Jiang, W., MA, Y. : Transparent Transmission-selective RADARInfrared Bi-Stealth Structure. Optics Express, 26(13) (2018).

12. Veselago, V. G.: The Electrodynamics of Substances with Simultaneously Negative Values of $\varepsilon$ and $\mu$. Soviet Physics Uspekhi. 10, 509-514 (1968)

13. Trang, P., Nguyen, B., Tiep, H., Thuy, L., Tung, N.T.: Symmetry-Breaking Metamaterials Enabling Broadband negative permeability. Journal of Electronic Materials. 45(5), 2547-2552 (2016)

14. RuoPeng, L., ZhiYa, Z., ChunLin, J., Tian, v.: Metamaterials beyond negative refractive index: Applications in telecommunication and sensing. Science China Press and Springer-Verlag Berlin Heidelberg. 59(7), 1007-1011 (2016)

15. Marquez, R., Medina, F., Raffi, R.: Role of Bianisotropy in Negative Permeability and Left-handed Metamaterials. Physical Review B. 65(14), 14440 (2002)

16. Lin, Y., Yan, K., Yao, D., Yu, Y.: Investigation of Electromagnetic Response of Terahertz Metamaterial by using Split-Disk Resonator. Optics and Laser Technology, Elsevier Ltd. 11, 509-514 (2006)

17. Caloz, C., Itoh, T.: Electromagnetic metamaterials. Transmission Line Theory and Microwave Applications, Wiley-Interscience (2006)

18. Marqués, R., Mesa, F., Martel, J., Medina, F. : Comparative analysis of edge- and broadside-coupled split ring resonators for metamaterial design-Theory and experiment. IEEE Trans. Antennas Propagation. 51(10), 2572-2581 (2003) 
19. Smith, D.R., Vier, C., Koschyl, T., Soukoulis, C. M.: Electromagnetic Parameter Retrieval from Inhomogeneous Metamaterials. Physical Review E-Statistical, Non Linear and Soft Matter Physics, American Physical Society. 71(3) (2005)

20. Xuan, B., et al.: Ultra-subwavelength thickness for dual/triple-band metamaterial absorber at very low frequency. Scientific Reports, 8, Article DOI: 10.1038/s41598-018-29896-4 (2018) 